\title{
Is hyperglycemia bad for the heart during acute ischemia?
}

\author{
Louis M. Chu, MD, ${ }^{\mathrm{a}}$ Robert M. Osipov, MD, ${ }^{\mathrm{a}}$ Michael P. Robich, MD, ${ }^{\mathrm{a}}$ Jun Feng, MD, PhD, ${ }^{\mathrm{a}}$ \\ Shizu Oyamada, MD, ${ }^{\mathrm{a}}$ Cesario Bianchi, MD, PhD, ${ }^{\mathrm{a}}$ and Frank W. Sellke, MD, FAHA, FACS ${ }^{\mathrm{a}, \mathrm{b}}$
}

Objective: This study investigates the impact of diabetes on myocardium in the setting of acute ischemiareperfusion in a porcine model.

\begin{abstract}
Methods: In normoglycemic (ND group) and alloxan-induced diabetic (DM group) male Yucatan pigs, the left anterior descending coronary artery territory was made ischemic and then reperfused. Hemodynamic values and myocardial function were measured. Monastryl blue and triphenyl tetrazolium chloride staining were used to assess size of the areas at risk and infarction. Glycogen content was assessed using periodic acid-Schiff staining. Cell death and survival signaling pathways were assessed by immunoblotting.
\end{abstract}

Results: Mean arterial pressure and developed left ventricular pressure were lower in the DM group $(P<.05)$. Whereas global left ventricular function was worse in the DM group $(P<.05)$, regional function in the area at risk was improved on the horizontal axis $(P<.05)$. Mean infarct size was smaller in the DM versus the ND group $(19 \%$ vs $43 \% ; P<.05)$, whereas the area at risk was similar in both groups $(34 \%$ vs $36 \% ; P=.7)$. Ischemic myocardium in the DM group displayed more prominent staining for glycogen compared with the ND group. In the area at risk, expression of cell survival proteins including phosphorylated endothelial nitric oxide synthase $(0.17 \pm 0.04$ vs $0.04 \pm 0.01 ; P<.05)$, heat shock protein $27(0.7 \pm 0.2$ vs $0.3 \pm 0.1 ; P<.05)$, nuclear factor $-\kappa \mathrm{B}$ $(0.14 \pm 0.02$ vs $0.03 \pm 0.01 ; P<.05)$, and mammalian target of rapamycin $(0.35 \pm 0.05$ vs $0.15 \pm 0.02 ; P<.05)$ were higher in DM animals, whereas in nonischemic tissue, expression of these proteins was similar or lower in the DM group.

Conclusions: Although type I diabetes worsens global left ventricular function, it is protective in the ischemic area, leading to increased expression of cell survival proteins and decreased infarct size. (J Thorac Cardiovasc Surg 2010;140:1345-52)

In the United States, more than 400,000 deaths per year are attributed to acute myocardial infarction (AMI). ${ }^{1}$ Current treatment of AMI is based on rapid restoration of blood flow to the ischemic area. Unfortunately, this results in additional reperfusion injury to the myocardium via an induced inflammatory response and the release of damaging free radicals. ${ }^{2,3}$ When added to the initial ischemic insult, reperfusion injury increases the incidence of a number of complications, including cardiac dysrhythmias, ${ }^{4}$ myocardial stunning, ${ }^{5}$ and coronary microvascular dysfunction. ${ }^{6}$ Ische-

From the Department of Surgery, ${ }^{\text {a }}$ Beth Israel Deaconess Medical Center, Harvard Medical School, Boston, Mass; and the Division of Cardiothoracic Surgery, ${ }^{b}$ Rhode Island Hospital, Alpert Medical School of Brown University, Providence, RI.

Funding for this project was provided to F.W.S by the National Heart, Lung, and Blood Institute (RO1 HL46716, HL69024, and HL85647), Capstone Corp, the National Institutes of Health T32-HL076130 (R.M.O, M.P.R), and the Irving Bard Memorial Fellowship (R.M.O, L.M.C, M.P.R)

Disclosures: Dr Frank W. Sellke has research support from Ikaria (Clinton, NJ), Capstone (Tempe, Ariz), and Ikaria Pharmaceutical (Seattle, Wash), and he is a consultant for Novo Nordisk (Princeton, NJ), Pfizer, and Cubist Pharmaceuticals (Lexington, Mass).

Received for publication Nov 10, 2009; revisions received March 11, 2010; accepted for publication May 18, 2010; available ahead of print June 14, 2010.

Address for reprints: Frank W. Sellke, MD, FAHA, FACS, Division of Cardiothoracic

Surgery, Rhode Island Hospital, Alpert Medical School at Brown University,

2 Dudley St, Ste 500, Providence, RI 02905. (E-mail: fsellke@lifespan.org).

$0022-5223 / \$ 36.00$

Copyright (C) 2010 by The American Association for Thoracic Surgery

doi:10.1016/j.jtcvs.2010.05.009 mia-reperfusion (I-R) injury results in a combination of necrosis, apoptosis, and autophagy of cardiac myocytes, the severity and onset of which can be modulated by manipulation of certain biochemical pathways via preconditioning and postconditioning. ${ }^{7}$ However, the mechanisms by which these pathways protect myocytes are incompletely understood.

On the other hand, it is widely known that diabetes mellitus is associated with increased incidence of multivessel coronary artery disease, congestive heart failure, and AMI. Both patients with type 1 and those with type 2 diabetes have significantly poorer clinical outcomes after AMI, with higher rates of repeat intervention, residual ventricular dysfunction, and overall mortality. ${ }^{8,9}$ Surprisingly, experimental studies looking at the sensitivity of the diabetic heart to ischemic injury have been divided, with studies showing both injurious and cardioprotective effects of diabetes. ${ }^{10}$ The majority of these studies, however, used either small animal models or in vitro models that do not adequately represent the biochemical and metabolic context that would be seen in patients with acute ischemia. In this study, we used a clinically relevant swine model of type I diabetes to explore the sensitivity of the diabetic heart to $\mathrm{I}-\mathrm{R}$ injury in vivo. We found that type I diabetes is cardioprotective against the insult of acute $\mathrm{I}-\mathrm{R}$ injury and that hyperglycemia may actually be beneficial to the heart in this setting. 


\begin{tabular}{|c|c|}
\hline \multicolumn{2}{|c|}{ Abbreviations and Acronyms } \\
\hline AAR & $=$ area at risk \\
\hline AMI & $=$ acute myocardial infarction \\
\hline AMPK & $\begin{aligned}= & \text { adenosine monophosphage- } \\
& \text { activated protein kinase }\end{aligned}$ \\
\hline $\mathrm{dP} / \mathrm{dt}$ & $\begin{aligned}= & \text { positive first derivative of left } \\
& \text { ventricular pressure }\end{aligned}$ \\
\hline 4EBP1 & $=4 \mathrm{E}$-binding protein 1 \\
\hline Erk & $=$ extracellularly regulated kinase \\
\hline HSP & $=$ heat shock protein \\
\hline $\mathrm{I}-\mathrm{R}$ & $=$ ischemia-reperfusion \\
\hline LAD & $\begin{array}{l}=\text { left anterior descending coronary } \\
\text { artery }\end{array}$ \\
\hline LV & $=$ left ventricular \\
\hline mTOR & $=$ mammalian target of rapamycin \\
\hline $\mathrm{NF}-\kappa \mathrm{B}$ & $=$ nuclear factor $-\kappa \mathrm{B}$ \\
\hline $\begin{array}{l}\text { PDK1 } \\
\text { phospho- } \\
\text { eNOS }\end{array}$ & $\begin{aligned}= & \text { pyruvate dehydrogenase kinase } 1 \\
= & \text { phosphorylated endothelial nitric } \\
& \text { oxide synthase }\end{aligned}$ \\
\hline SAPK/ & $=$ stress-activated protein kinase/c-Jun \\
\hline JNK & NH2-terminal kinase \\
\hline
\end{tabular}

\section{MATERIALS AND METHODS Experimental Design}

Sixteen intact male Yucatan mini-swine were divided into 2 groups: nondiabetic (ND, $\mathrm{n}=8)$ and diabetic (DM, $\mathrm{n}=8)$. All animals were fed normal chow (S11 Purina, St Louis, Mo). Diabetes was induced in DM animals by a single intravenous injection of alloxan $(200 \mathrm{mg} / \mathrm{kg}$ ) at age 15 weeks (Sinclair Research Center, Inc, Columbia, Mo). Alloxan-treated animals that maintained blood glucose levels greater than $250 \mathrm{mg} / \mathrm{dL}$ were used in the DM group. At 20 weeks of age (5 weeks of exposure to diabetes), animals were subjected to acute ischemia by occluding the left anterior descending coronary artery (LAD) for 60 minutes, followed by release of the LAD and reperfusion for 120 minutes. Hemodynamic and functional measurements were taken at baseline and for every 30 minutes thereafter. Myocardial segmental shortening in the longitudinal axis (parallel to the LAD) and horizontal axis (perpendicular to the LAD) were recorded as well. At the completion of the protocol, the heart was excised, and tissue samples were collected for molecular analyses as described below.

\section{Animals \\ Swine were housed individually and provided with normal chow and wa- ter ad libitum. All experiments were approved by the Beth Israel Deaconess Medical Center Institutional Animal Care and Use Committee and con- formed to the US National Institutes of Health guidelines regulating the care and use of laboratory animals (NIH publication No. 5377-3, 1996).}

\section{Surgical Protocol}

Swine at age 20 weeks were sedated with tiletamine/zolazepam (Telazol; $1.5 \mathrm{mg} / \mathrm{kg}$, intramuscular) and weighed before endotracheal intubation and ventilation with a volume-cycled ventilator (North American Drager, Telford, Pa). Anesthesia was maintained with 2.0\% isoflurane (Abbott Laboratories, Inc, North Chicago, Ill). A $5 \mathrm{~F}$ arterial sheath was passed into the right femoral artery via direct cutdown and used for arterial blood sampling and arterial blood pressure monitoring. Arterial blood gas, hematocrit, and core temperature were measured at the start of surgery and every 30 minutes thereafter. Each animal received a 1-L bolus of lactated Ringer solution followed by continuous infusion $\left(15 \mathrm{~mL} \cdot \mathrm{kg}^{-1}\right.$ - $\left.\mathrm{h}^{-1}\right)$. A phenylephrine drip $\left(0.25 \mu \mathrm{g} \cdot \mathrm{kg}^{-1} \cdot \mathrm{min}^{-1}\right)$ to prevent isoflurane-induced hypotension, heparin ( 80 units $/ \mathrm{kg}$ bolus), and lidocaine ( $1.5 \mathrm{mg} / \mathrm{kg}$ bolus) to prevent ventricular dysrhythmia were administered. A median sternotomy was performed. A catheter-tipped manometer (Millar Instruments, Inc, Houston, Tex) was introduced through the apex of the heart to record left ventricular (LV) pressure. Segmental shortening in the ischemic area at risk (AAR) was assessed with a digital ultrasonic crystal measurement system (Sonometrics Corp, London, Ontario, Canada) using four 2-mm digital ultrasonic probes implanted in the subepicardial layer approximately $10 \mathrm{~mm}$ apart within the AAR. Cardiosoft software (Sonometrics Corp) was used for functional measurements. LAD flow was monitored by a Doppler probe (Transonic Systems, Inc, Ithaca, NY). The LAD was occluded $3 \mathrm{~mm}$ distal to the origin of the second diagonal branch by a Rommel tourniquet. After 60 minutes, the tourniquet was released and the myocardium allowed to reperfuse for 120 minutes. The LAD was then religated, the ascending aorta was crossclamped, and monastryl blue pigment (Engelhard Corp, Louisville, Ky) was injected into the aortic root to demarcate the AAR. The heart was excised and sectioned into three $1 \mathrm{~cm}$-thick slices perpendicular to the LAD from the apex to the point of ligation. Tissue from the slice $1 \mathrm{~cm}$ proximal to the apex was used in molecular studies. The remaining tissue was incubated in $1 \%$ triphenyl tetrazolium chloride [(TTC; Sigma Chemical Co, St. Louis, Mo] solution for 30 minutes and infarct size was assessed as described below. Ventricular fibrillation or ventricular tachycardia events were treated with an extra dose of lidocaine $(1.5 \mathrm{mg} / \mathrm{kg})$ and electrical cardioversion with 20 to $50 \mathrm{~J}$ for persistent dysrhythmias.

\section{Measurement of Global and Regional Myocardial Function}

Indices of global and regional myocardial function were monitored during the entire experiment: mean arterial pressure, developed LV pressure, positive first derivative of $\mathrm{LV}$ pressure $(\mathrm{dP} / \mathrm{dt})$, and longitudinal and horizontal segmental shortening in the AAR. These indices were recorded for 10 sequential beats, at baseline, and then every 30 minutes thereafter using the Sonometrics Cardiosoft system as previously described. ${ }^{11}$

\section{Quantification of Myocardial Infarct Size}

The LV (including septum) was isolated, cut into 1-cm slices, and immediately immersed in $1 \%$ triphenyl tetrazolium chloride in phosphatebuffered saline (Boston Bioproducts, Worcester, Mass) at $38^{\circ} \mathrm{C}$ for $30 \mathrm{~min}-$ utes. The infarct area (characterized by absence of staining), noninfarcted AAR (characterized by bright red tissue staining), and the nonischemic ventricle (characterized by purple tissue staining) were photographed and measured. AAR as a percentage of total LV surface area and percent infarction in the AAR was calculated in each individual slice by planimetry (Image $\mathbf{J}$ 1.4) using the following equations:

$$
\text { AAR size }=(\text { AAR surface area } / \text { LV total surface area }) \times 100
$$

Infarct size $=($ LV infarct surface area $/$ LV AAR surface area $) \times 100$

\section{Glycogen Staining}

Sections of ischemic and nonischemic myocardium from ND $(n=5)$ and $\mathrm{DM}(\mathrm{n}=7)$ animals were placed immediately into $10 \%$ formalin and subjected to periodic acid-Schiff staining to assess glycogen content, with amylase-treated sections serving as negative controls. Ischemic areas were evaluated for amount of glycogen by a pathologist in a blinded fashion and were assigned a score from 0 to 5 , with 0 representing complete absence of glycogen and 5 representing strongest staining for glycogen (nonischemic myocardium). 


\section{Western Blotting}

Myocardial samples were homogenized in RIPA buffer (Boston BioProducts, Inc, Worcester, Mass) and total protein concentration determined by bicinchoninic acid assay (BCA assay; Pierce, Rockford, Ill). Equal amounts of protein $(40 \mu \mathrm{g})$ were subjected to sodium dodecyl sulfate polyacrylamide gel electrophoresis and immunoblotting as previously described. ${ }^{11}$ Primary antibodies were used according to the manufacturer's recommendation. Levels of Akt, phospho-Akt (Thr308), phospho-Akt (Ser473), phosphorylated endothelial nitric oxide synthase (Ser1177) (phospho-eNOS), extracellularly regulated kinase (Erk) $1 / 2$, phospho-Erk $1 / 2$ (Thr202/Tyr204)), mammalian target of rapamycin (mTOR), phosphomTOR (Ser2448), stress-activated protein kinase/c-Jun NH2-terminal kinase (SAPK/JNK), p70S6K1, phospho-p70S6K1 (Thr389), 4E-binding protein 1 (4EBP1), and phospho-4EBP1 (Thr37/46), nuclear factor $-\kappa \mathrm{B}$ (NF- $\kappa$ B) p65 and (NF-phospho-NF- $\kappa$ B p65 (Ser536), pyruvate dehydrogenase kinase 1 (PDK1), and phospho-PDK1 (Ser241) (Cell Signaling Technology, Beverly, Mass), heat shock protein 27 (HSP27), and HSP70 (Stressgen, Ann Arbor, Mich) were assessed. Myocardial samples from the nonischemic and the ischemic territories were assessed separately. Vinculin was used to confirm equal protein loading, and band intensities were normalized to Ponceau staining (Sigma Chemical Co).

\section{Statistical Analysis}

Clinical, hemodynamic, and global and regional LV functional data were analyzed using 2-way repeated-measures analysis of variance (Systat, San Jose, Calif). Post hoc multiple comparison Student-Newman-Keuls test was applied. Myocardial infarct size, glycogen staining, and Western blot densitometry were analyzed by the unpaired Student $t$ test. Western blot data are presented as density in arbitrary units. Data are reported as mean \pm standard error of the mean.

\section{RESULTS}

\section{Diabetic Swine}

There was a significant difference in animal weight between ND and DM animals (ND $22.2 \pm 1 \mathrm{~kg}$ vs DM $17 \pm$ $1 \mathrm{~kg} ; P=.004)$. The average blood glucose level was significantly higher in the DM group versus the ND group (309 \pm $57 \mathrm{mg} / \mathrm{dL}$ vs $37 \pm 10 \mathrm{mg} / \mathrm{dL} ; P<.01)$. There were no significant differences between groups with respect to arterial blood gas measurements, hematocrit, and core temperature at any time.

\section{Myocardial Infarct Size}

The size of the AAR was not significantly different between groups $(34 \%$ in DM vs $36 \%$ in ND; $P=.7$; Figure 1,A), whereas the size of the infarct area was smaller in the DM group as compared with the ND group $(19 \%$ in DM vs $43 \%$ in ND; $P<.05$; Figure $1, B)$.

\section{Hemodynamic Parameters}

Before LAD occlusion, the heart rate $(P<.01)$ and developed LV pressure $(P<.01$ were lower in the DM group as compared with the ND group, whereas mean arterial pressure was slightly higher $(P=.3)$. After occlusion, all 3 hemodynamic parameters were lower in the DM group. LAD blood flow during the reperfusion period was lower in the DM group $(P<.05$; Figure 2$)$.

\section{Global and Regional Myocardial Function}

Global systolic LV function as determined from $+\mathrm{dP} / \mathrm{dt}$ was significantly worse in the DM group as compared with the ND group at all times $(P<.01$; Figure $2, E)$. Regional LV function as assessed by percent segmental shortening on the horizontal axis was significantly better in the DM group as compared with the ND group (Figure 2, F; $P<.05)$, whereas function on the longitudinal axis was not significantly different between groups (not shown).

\section{Glycogen Staining}

In the AAR, glycogen content was higher in the DM group compared with the ND group $(1.36 \pm 0.14$ vs $0.70 \pm 0.20 ; P=.02$; Figure 3).

\section{Protein Expression in the Nonischemic Territory} Cell survival signaling. The expression of total Akt was higher $(P<.01)$ in the ND group as compared with the DM group, whereas the expression of phospho-Akt (Ser473) and phospho-Akt (Thr308) was similar between groups (not shown). The ratio of phospho-Akt (Thr 308) to total Akt was significantly higher $(P<.01)$ in the DM group compared with the ND group (Figure 4, $A$ ). The expression of PDK1 was lower $(P<.01)$ in the DM group as compared with the ND group (Figure 4, $B$ ), whereas the expression of phospho-PDK1 was similar between groups (ND $0.1 \pm 0.01$, DM $0.09 \pm 0.01 ; P=.1$ ). The expression of HSP27 was similar $(P=.3$ ) between groups (Figure 4, $C$ ), as was the expression of HSP70 $(P=.6$; Figure $4, D)$. The expression of phospho-eNOS (Ser1177) tended to be lower in the DM group as compared with the ND group (Figure 4, E).

The expression of mTOR, phospho-NF- $\kappa \mathrm{B}$ p65 (Figure 4, $F$ ), phospho-mTOR (Ser2448) (Figure 4, $G$ ), phospho-4EBP1 (Thr37/46) (Figure 4, I), NF- $\kappa \mathrm{B}$ p65, p70S6K, and 4E-BP1 (not shown), was similar between the groups. Phospho-p70S6K (Thr389) and phospho-NF- $\kappa$ B p65 (Ser536) were not detected in either group.

Mitogen-activated protein kinase signaling. The expression of total Erk $1 / 2$ was similar $(P=.9)$ between the groups, whereas the expression of phospho-Erk 1/2 (Thr202/ Tyr204) was lower in the DM group as compared with the ND group (DM $0.03 \pm 0.01$, ND $0.07 \pm 0.02 ; P=.04$ ). The expression of phospho-p90RSK (Ser308) was similar $(P=.4)$ between the 2 groups (Figure 5, $B)$. The expression of SAPK/JNK was lower $(P=.03)$ in the DM group (Figure 5, C).

\section{Protein Expression in the AAR}

Cell survival signaling. The expression of total Akt and phospho-Akt (Ser473), and phospho-Akt (Ser 473) was similar between the 2 groups (not shown), whereas the ratio of phospho-Akt (Thr308) to total Akt tended to be higher in the 

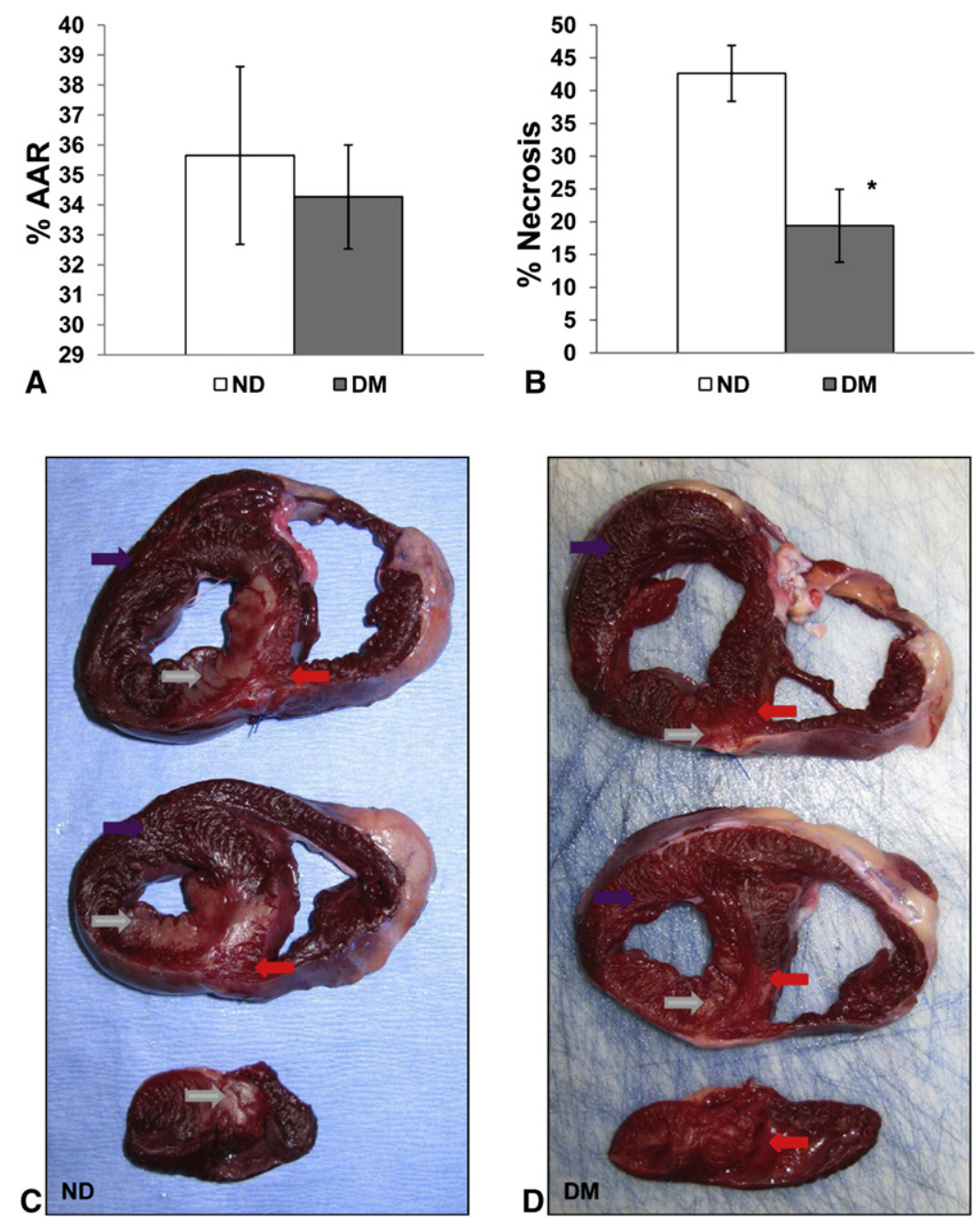

FIGURE 1. Size of AAR as a percentage of LV surface area $(P=.7, \mathrm{~A})$ and necrotic area as a percentage of AAR (B) in ND and DM animals. Shown are representative slices of ND (C) and DM (D) ventricle, with purple arrows pointing to nonischemic area, red arrows to AAR, and gray arrows to infarct area. Data presented as mean \pm SEM comparing ND $(\mathrm{n}=8)$ and DM $(\mathrm{n}=8)$ groups. ${ }^{*} P<.05$. AAR, Area at risk; $L V$, left ventricular; $N D$, nondiabetic; $D M$, diabetic.

DM group as compared with the ND group (Figure $4, A$ ). The expression of PDK1 $(P<.01$; Figure $4, B)$ and phospho-PDK1 (Ser241) (DM $0.2 \pm 0.01$, ND $0.08 \pm$ $0.02 ; P<.01)$ was higher in the DM group. The expression of HSP27 and HSP70 was higher $(P<.05)$ in the DM group, as shown in Figure 4, $C$ and $D$. The expression of phosphoeNOS (Ser1177) was higher $(P<.01)$ in the DM group (Figure 4, E). The expression of mTOR (DM $0.035 \pm$ 0.005 , ND $0.017 \pm 0.002 ; P<.01)$ and phospho-mTOR (Ser2448) (Figure $4, G$ ) was higher in the DM group. The expressions of p70S6K (DM $0.09 \pm 0.01$, ND $0.05 \pm$ $0.02 ; P<.01)$ and phospho-p70S6K $($ Thr389) $(P<.02$; Figure $4, H$ ) were higher in the DM group. The expression of phospho-4E-BP1 $(\mathrm{Thr} 37 / 46)$ was higher $(P<.01)$ in the DM group (Figure $4, I$ ), whereas the expression of phospho-4E-BP1 tended to be higher (DM $0.3 \pm 0.01$, ND $0.2 \pm 0.02 ; P=.07)$ in the same group. The expression of
NF- $\kappa$ B p65 (DM $0.14 \pm 0.02$, ND $0.03 \pm 0.01 ; P<.01)$ and phospho-NF- $\kappa$ B p65 (Ser536) (Figure $4, F$ ) was also higher in the DM group.

Mitogen-activated protein kinase signaling. Total Erk 1/ 2 was more highly expressed in ND animals than DM animals (ND $0.09 \pm 0.01$, DM $0.04 \pm 0.01 ; P=.01$ ), but the ratio of phospho-Erk 1/2 (Thr202/Tyr204) expression to total Erk $1 / 2$ was higher $(P=.01)$ in the DM group (Figure 5, $A)$. The expression of phospho-p90RSK (Ser308) was higher $(P=.01)$ in the DM group (Figure $5, B)$. The expression of SAPK/JNK was also higher $(P<.05)$ in the DM group (Figure 5, C).

\section{DISCUSSION}

In this study, we examined the effects of type 1 diabetes on LV I-R injury. $\mathrm{We}^{11}$ previously reported that I-R injury is increased in a porcine hypercholesterolemic model and 

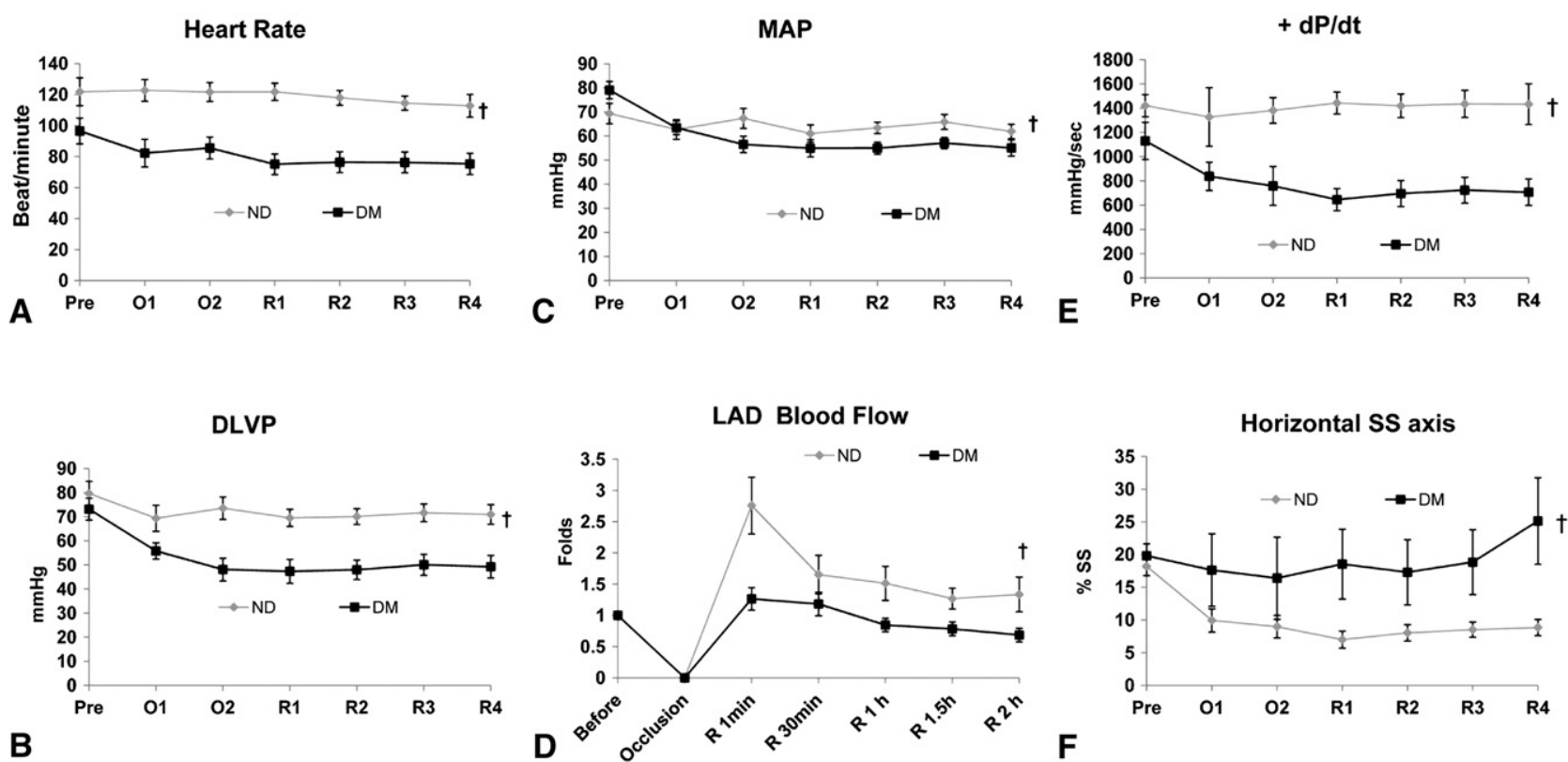

FIGURE 2. Hemodynamic and functional data. Heart rate (A), developed left ventricular pressure ( $D L V P, \mathrm{~B})$, mean arterial pressure (MAP, C), left anterior descending $(L A D)$ artery blood flow (D), first derivative of LV pressure over time $(+d P / d t, \mathrm{E})$, and horizontal segmental shortening $(S S)$ axis $(\mathrm{F})$. Pre $=$ baseline, $O 1=30$ minutes occlusion, $O 2=60$ minutes occlusion, $R 1=30$ minutes reperfusion, $R 2=60$ minutes reperfusion, $R 3=90$ minutes reperfusion, $R 4=120$ minutes reperfusion. Data presented as mean \pm SEM comparing ND $(\mathrm{n}=8)$ and DM $(\mathrm{n}=8)$ groups. $\dagger P<.05$.

suspected that similar findings would be evident in our type 1 diabetic model. To our surprise, this study demonstrates that experimental type I diabetes is cardioprotective against acute I-R injury, limiting infarct size, improving regional function, and increasing expression of cell survival mole- cules by at-risk myocytes in a porcine model of myocardial infarction.

It is widely documented that patients with type 1 diabetes have worse outcomes after coronary artery bypass grafting than do nondiabetic patients. However, several studies
A

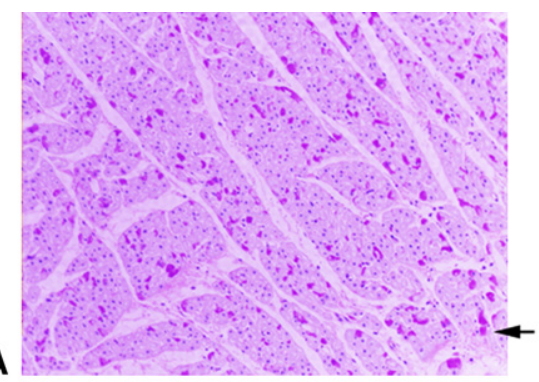

C

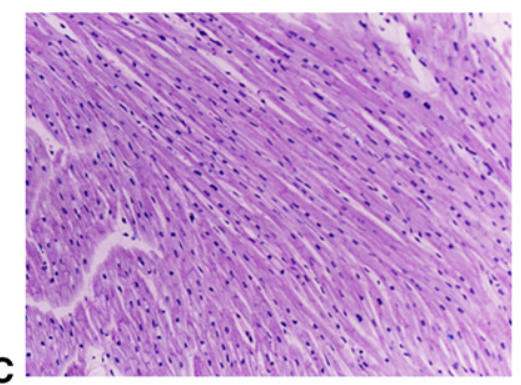

B

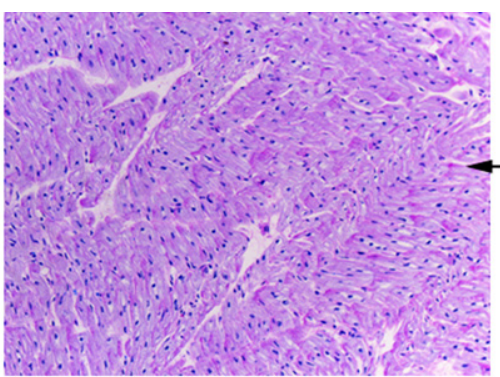

Glycogen Staining in Ischemic Territory

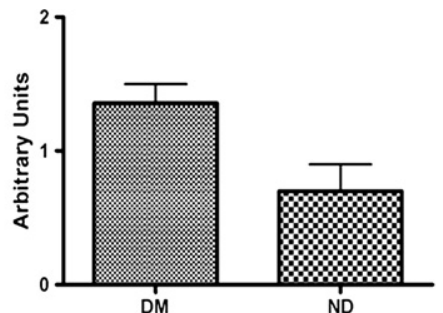

FIGURE 3. Glycogen staining using period acid-Schiff stain in nonischemic DM (A), ischemic DM (B), and ischemic ND (C) myocardium, with black arrows indicating intracellular glycogen. Sections shown are representative of all sections assessed. DM $(n=7)$ and ND $(n=5)$ sections were scored in a blinded fashion and glycogen content was compared (D). Data presented as mean \pm SEM in arbitrary units. $P=.02$. $D M$, Diabetic; $N D$, nondiabetic. 


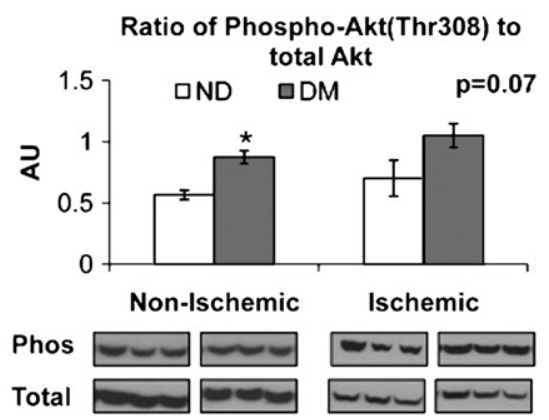

A

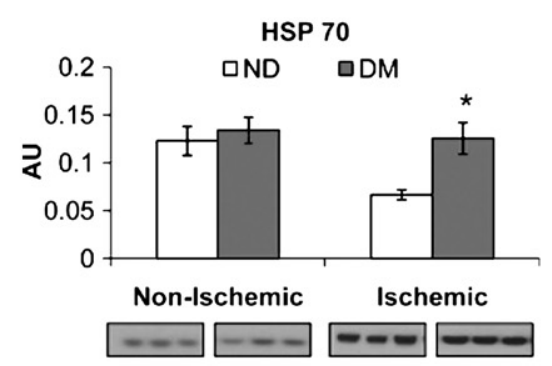

D

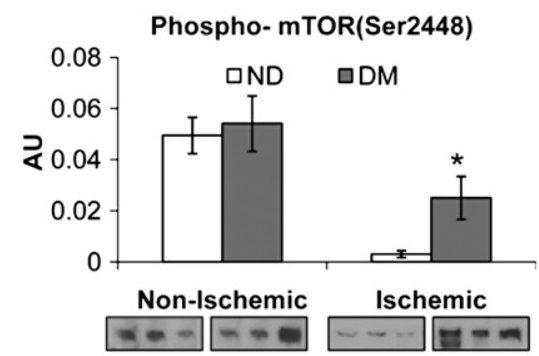

G

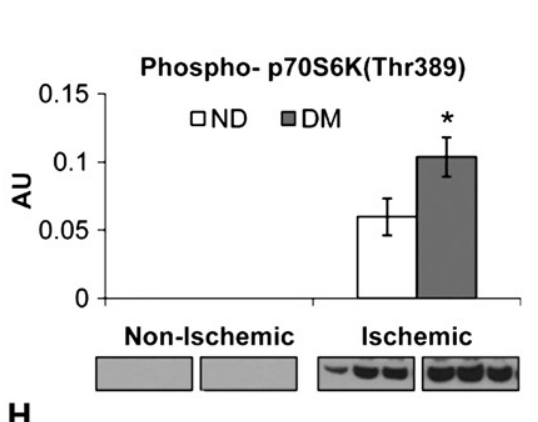

H

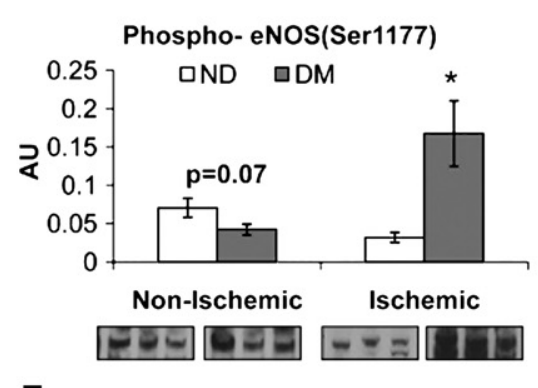

E

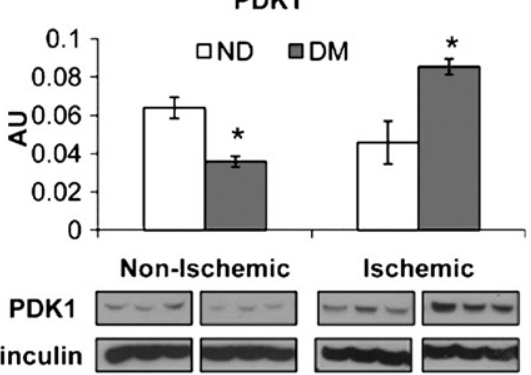

B

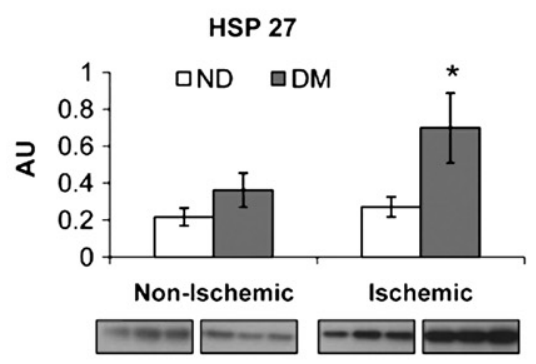

C

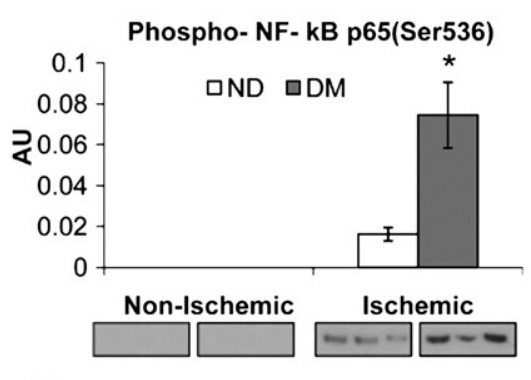

$\mathbf{F}$

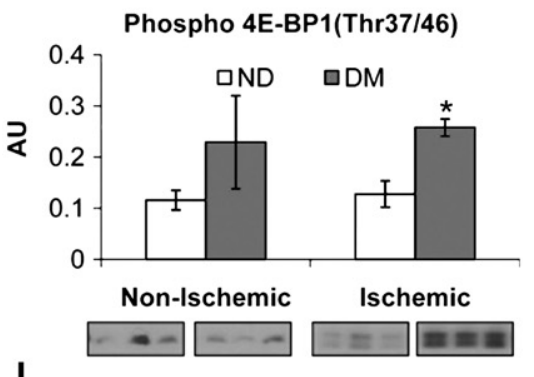

FIGURE 4. Selected cell-survival protein immunblotting results. Ratio of phospho-Akt to total Akt (A), PDK1 (B), HSP27 (C), HSP70 (D), phospho-eNOS (E), phospho-NF- $\kappa$ B $(\mathrm{F})$, phospho-mTOR $(\mathrm{G})$, phospho-p70S6K $(\mathrm{H})$, and phospho-4E-BP1 (I) expression was assessed in the ischemic and nonischemic territories of ND $(n=8)$ and DM $(n=8)$ animals. Representative bands are shown, with density expressed as arbitrary units. Data presented as mean \pm SEM in arbitrary units $(\mathrm{AU}) .{ }^{*} P<.05 . P D K 1$, Pyruvate dehydrogenase kinase $1 ; H S P$, heat shock protein; eNOS, endothelial nitric oxide synthase; $m T O R$, mammalian target of rapamycin; $4 E B P 1$, 4E-binding protein $1 ; N D$, nondiabetic; $D M$, diabetic.

have shown that the diabetic heart is actually less sensitive to ischemic injury, especially in in vivo models. Chen and associates $^{12}$ showed that type I diabetic rat hearts demonstrated enhanced tolerance to I-R that was abolished by preoperative treatment with insulin. Ma and colleagues ${ }^{13}$ found that capillary density and the expression of cardioprotective proteins, including vascular endothelial growth factor, eNOS, and phospho-Akt, were increased in rats exposed to 2 weeks of diabetes compared with nondiabetic rats, whereas infarct size and caspase-3, a proapoptotic signal, were decreased.

\section{Diabetic Swine}

Our DM swine achieved fasting blood glucose levels nearly 9 times that of ND swine, demonstrating successful induction of type 1 diabetes with alloxan. The mean fasting blood glucose level in our ND swine of $37 \mathrm{mg} / \mathrm{dL}$ was similar to previously reported values for nondiabetic Yucatan swine $(43 \mathrm{mg} / \mathrm{dL}) .{ }^{14}$

Diabetes decreases infarct size and improves regional function. The most striking finding in this study was the significant decrease in infarct size seen in DM compared with ND animals. A possible explanation lies in the regulation of myocardial glucose uptake during cardiac stress. Under resting conditions, metabolism of fatty acids yields $60 \%$ to $70 \%$ of the adenosine triphosphate requirement of the heart, but during situations of stress the energy preference shifts to favor use of glucose, diminishing the release of damaging free radicals by fatty acid oxidation. ${ }^{15}$ It was thought that, in the insulin-deficient environment of type I 


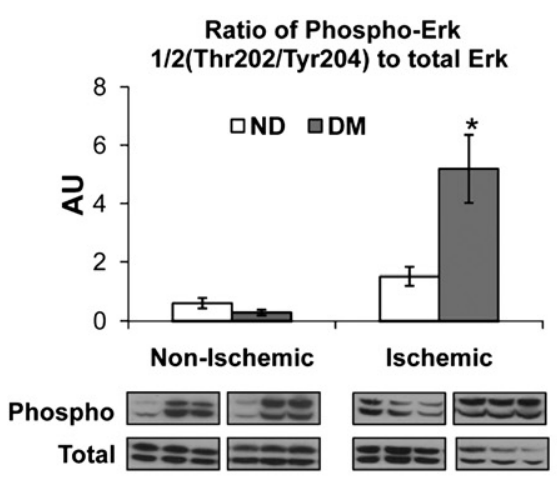

A

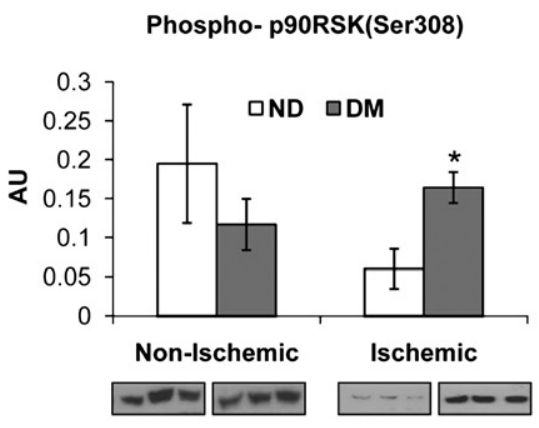

B
SAPK /JNK

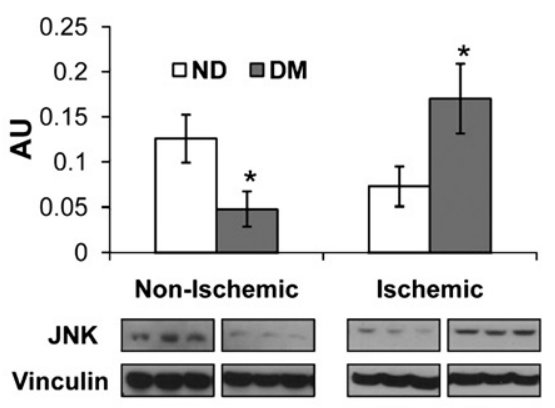

C

FIGURE 5. Selected mitogen activated protein kinase immunoblotting results. Ratio of phospho-Erk to total Erk (A), phospho-p90RSK (B), and SAPK/JNK (C) expression was assessed in the ischemic and nonischemic territories of ND $(\mathrm{n}=8)$ and DM (n $=8)$ animals. Representative bands are shown, with density expressed as arbitrary units. Data presented as mean $\pm \mathrm{SEM}$ in arbitrary units $(A U)$. ${ }^{*} P<.05$. Erk, Extracellularly regulated kinase; $S A P K / J N K$, stressactivated protein kinase/c-Jun NH2-terminal kinase; $N D$, nondiabetic; $D M$, diabetic.

diabetes, impaired glucose uptake led to less efficient myocardial metabolism under ischemic conditions. However, recent studies have uncovered alternative pathways that may take over glucose regulation during situations of stress. Adenosine monophosphate-activated protein kinase (AMPK) has been shown to be an important effector of glycolysis and glucose uptake during cellular stress. In the myocardium, AMPK promotes the translocation of glucose transporter 4 to the cell membrane, increasing myocardial glucose uptake and protecting the heart from ischemia. ${ }^{16}$ Hypoxia-induced factor- $1 \alpha$ has also been found to play a role in glucose metabolism under stress, increasing expression of glucose transporter 1 and enzymes involved in glycolysis. ${ }^{17}$ Hyperglycemic, insulin-deficient animals likely use these noninsulin-dependent pathways to more efficiently metabolize glucose, thus decreasing ischemic damage. The improvement in horizontal contractility seen in the AAR of our DM hearts could be due to this increased availability of metabolic substrate for myocardial contraction. It should be noted that blood flow through the LAD during reperfusion was lower in the DM group, which could mean decreased delivery of inflammatory mediators and free radicals and decreased reperfusion injury. This difference between groups is one limitation of the current study, it but does not explain our Western blotting results.

Diabetic hearts display worsened global function. Mean arterial pressure, developed LV pressure, and $\mathrm{dP} / \mathrm{dt}$ were all lower in DM animals, reflecting worsened global LV function compared with ND animals. Chronic diabetes, through a variety of mechanisms including the accumulation of glycolytic intermediates, the production of toxic intermediates from free fatty acid metabolism, and increased myocardial fibrosis, leads to cellular, structural, and functional changes in the myocardium termed diabetic cardiomyopathy. ${ }^{18}$ In contrast to the AAR, where myocardial stress leads to increased glucose use, the nonischemic diabetic LV may preferentially metabolize fatty acids, leading to increased oxidative stress and worsened global LV function.

Glycogen stores in AAR are increased in diabetic hearts. Glycogen staining of myocardium from the AAR revealed a higher amount of glycogen in DM animals compared with ND animals after I-R. This finding could be interpreted as either decreased use or increased availability of glycogen, but given our finding of decreased infarct size in the DM group, the latter scenario is more likely. Previous studies have demonstrated that diabetic myocardium contains more glycogen than nondiabetic myocardium at baseline. ${ }^{19}$ During situations of stress, when glucose is the preferred metabolite, the excess glycogen in ischemic diabetic myocardium could fuel cardioprotective mechanisms, whereas the glycogen supply in ischemic nondiabetic myocardium is exhausted.

Increased expression of cell survival proteins in diabetic hearts. In nonischemic myocardium, the expression of several proteins involved in cell survival were either similar or lower in DM animals compared with controls. Interestingly, the expression of most of these same proteins in the ischemic area was significantly higher in the DM group compared with the control group, indicating that cell survival pathways in ischemic myocardium were more active in these animals. Akt, PDK1, and eNOS have been shown to reduce adenosine triphosphate breakdown and mitochondrial $\mathrm{Ca}^{+}$loading, ultimately preconditioning the myocardium against $\mathrm{I}-\mathrm{R}$ injury. ${ }^{7}$ HSP27 has been shown to protect against I-R injury via stabilization of troponin $\mathrm{I}$ and $\mathrm{T}^{20}$, and HSP70 has been found to play a role in late ischemic preconditioning as well. ${ }^{21}$ Phosphorylation and activation of NF- $\kappa \mathrm{B}$ is linked with cell survival and inflammation. ${ }^{22}$

Expression of mTOR, phospho-mTOR, phospho-4EBP1, and p70S6K, a kinase intermediate in the mTOR pathway, was also higher in the ischemic area of DM animals compared with controls. The glucose-dependent mTOR pathway 
is critical to protein translation, and inhibition of mTOR by rapamycin has been shown to block the protective effects of preconditioning. ${ }^{23}$ The increased glucose in DM animals likely means more available substrate for glucose-dependent mechanisms such as the mTOR pathway.

The same trend was seen when looking at the expression of mitogen-activated proteins involved in cell growth and survival. Erk 1/2, its downstream kinase p90RSK, ${ }^{24}$ and $\mathrm{SAPK} / \mathrm{JNK}^{25}$ have all been associated with preconditioning and cardioprotection from I-R injury. It is evident that a host of cell survival proteins and cell growth-related proteins are upregulated in the ischemic area of diabetic animals.

Duration of hyperglycemia. Several studies in small animal models have found that streptozotocin-induced diabetes exerts contradictory effects on myocardial ischemia in chronic versus acute hyperglycemia, reporting increased infarct size and worsened myocardial function in animals with long-standing disease. ${ }^{14}$ It is also widely known that longterm diabetes increases the incidence and complications of myocardial infarction and can progress to diabetic cardiomyopathy. A limitation of the current study is that it examines the effects of hyperglycemia at only 1 time point, relatively early in the course of diabetes (5 weeks). It is likely that, as the disease progresses, the other previously mentioned biological sequelae of hyperglycemia result in decreased resistance against acute ischemia.

\section{CONCLUSIONS}

Recently, government agencies such as the National Institutes of Health and the Food and Drug Administration have been putting greater emphasis on clinically relevant large animal models for preclinical experiments. This is due to the fact that although rodent models of I-R injury and other experiments may give a wealth of information, the findings often do not translate well to patients.

This study provides evidence that type 1 diabetes mellitus and resultant hyperglycemia, though detrimental to global cardiac function and associated with poorer prognosis, are actually cardioprotective against myocardial I-R injury in the short term. This is likely a result of increased availability and use of glucose, the heart's preferred energy substrate in times of stress. Thus, the current clinical practice of tightly controlling blood glucose in patients having cardiac events may be detrimental to the heart in the acute setting. Recent investigation into AMPK and hypoxia-induced factor- $1 \alpha$, potent regulators of glucose uptake in the myocardium, provides possible mechanisms for the cardioprotective properties of hyperglycemia. Additional studies using this model to assess the activity of these enzymes and their targets would help to further elucidate the pathway and possibly develop therapeutic modalities for protection against ischemia.

We thank Beth Israel Deaconess Medical Center Animal Research Facility and Core Histology Laboratory staff for their efforts.

\section{References}

1. Lloyd-Jones D, Adams R, Carnethon M, De Simone G, Ferguson TB, Flegal K, et al. Heart disease and stroke statistics-2009 update: a report from the American Heart Association Statistics Committee and Stroke Statistics Subcommittee. Circulation. 2009; 119:480-6.

2. Roberts MJ, Young IS, Trouton TG, Trimble ER, Khan MM, Webb SW, et al. Transient release of lipid peroxides after coronary artery balloon angioplasty. Lancet. 1990;336:143-5.

3. Kloner RA, Giacomelli F, Alker KJ, Hale SL, Matthews R, Bellows S. Influx of neutrophils into the walls of large epicardial coronary arteries in response to ischemia/reperfusion. Circulation. 1991;84:1758-72.

4. Manning AS, Hearse DJ. Reperfusion-induced arrhythmias: mechanisms and prevention. J Mol Cell Cardiol. 1984;16:497-518.

5. Ambrosio G, Tritto I. Clinical manifestations of myocardial stunning. Coron Artery Dis. 2001;12:357-61.

6. Lefer AM, Lefer DJ. The role of nitric oxide and cell adhesion molecules on the microcirculation in ischaemia-reperfusion. Cardiovasc Res. 1996;32:743-51.

7. Murphy E, Steenbergen C. Mechanisms underlying acute protection from cardiac ischemia-reperfusion injury. Physiol Rev. 2008;88:581-609.

8. Katayama T, Nakashima H, Takagi C, Honda Y, Suzuki S, Iwasaki Y, et al. Clinical outcomes and left ventricular function in diabetic patients with acute myocardial infarction treated by primary coronary angioplasty. Int Heart J. 2005;46: 607-18.

9. Fisher BM. Heart abnormalities in IDDM. Diabetologia. 1997;40(Suppl. 2): S127-9.

10. Fevray D, Lopaschuk GD. Controversies on the sensitivity of the diabetic heart to ischemic injury: the sensitivity of the diabetic heart to ischemic injury is decreased. Cardiovasc Res. 1997;34:113-20.

11. Osipov RM, Bianchi C, Feng J, Clements RT, Liu Y, Robich MP, et al. Effect of hypercholesterolemia on myocardial necrosis and apoptosis in the setting of ischemia-reperfusion. Circulation. 2009;120(11 Suppl):S22-30.

12. Chen H, Wu XJ, Lu XY, Zhu L, Wang LP, Yang HT, et al. Phosphorylated heat shock protein 27 is involved in enhanced heart tolerance to ischemia in short-term type 1 diabetic rats. Acta Pharmacol Sin. 2005;26:806-12.

13. Ma G, Al-Shabrawey M, Johnson JA, Datar R, Tawfik HE, Guo D, et al. Protection against myocardial ischemia/reperfusion injury by short-term diabetes: enhancement of VEGF formation, capillary density, and activation of cell survival signaling. Naunyn Schmiedebergs Arch Pharmacol. 2006;373:415-27.

14. Bellinger DA, Merricks EP, Nichols TC. Swine models of type 2 diabetes mellitus: insulin resistance, glucose tolerance, and cardiovascular complications. ILAR J. 2006;47:243-58.

15. Bertrand L, Horman S, Beauloye C, Vanoverschelde JL. Insulin signaling in the heart. Cardiovasc Res. 2008;79:238-48.

16. Russell RR 3rd, Li J, Coven DL, Pypaert M, Zechner C, Palmeri M, et al. AMPactivated protein kinase mediates ischemic glucose uptake and prevents postischemic cardiac dysfunction, apoptosis, and injury. J Clin Invest. 2004;114:495-503.

17. Bartrons R, Caro J. Hypoxia, glucose metabolism and the Warburg's effect. J Bioenerg Biomembr. 2007;39:223-9.

18. Khavandi K, Khavandi A, Asghar O, Greenstein A, Withers S, Heagerty AM, et al. Diabetic cardiomyopathy-a distinct disease? Best Pract Res Clin Endocrinol Metab. 2009;23:347-60.

19. Ravingerova T, Stetka R, Volkovova K, Pancza D, Dzurba A, Ziegelhöffer A, et al. Acute diabetes modulates response to ischemia in isolated rat heart. $\mathrm{Mol}$ Cell Biochem. 2000;210(1-2):143-51.

20. Lu XY, Chen NL, Cai XL, Yang HT. Overexpression of heat shock protein 27 protects against ischaemia/reperfusion-induced cardiac dysfunction via stabilization of troponin I and T. Cardiovasc Res. 2008;79:500-8.

21. Melling CW, Thorp DB, Milne KJ, Noble EG. Myocardial Hsp70 phosphorylation and PKC-mediated cardioprotection following exercise. Cell Stress Chaperones. 2009; 14:141-50.

22. Pereira SG, Oakley F. Nuclear factor-kappaB1: regulation and function. Int J Biochem Cell Biol. 2008;40:1425-30.

23. Khan S, Salloum F, Das A, Xi L, Vetrovec GW, Kukreja RC. Rapamycin confers preconditioning-like protection against ischemia-reperfusion injury in isolated mouse heart and cardiomyocytes. J Mol Cell Cardiol. 2006;41:256-64.

24. Ping P, Zhang J, Cao X, Li RC, Kong D, Tang XL, et al. PKC-dependent activation of $\mathrm{p} 44 / \mathrm{p} 42$ MAPKs during myocardial ischemia-reperfusion in conscious rabbits. Am J Physiol. 1999;276(5 Pt 2):H1468-81.

25. Shao Z, Bhattacharya K, Hsich E, Park L, Walters B, Germann U, et al. c-Jun $\mathrm{N}$-terminal kinases mediate reactivation of Akt and cardiomyocyte survival after hypoxic injury in vitro and in vivo. Circ Res. 2006;98:111-8. 